\title{
A Prospective Analysis of Plasma Adiponectin and Risk of Incident Cancer: The Dallas Heart Study
}

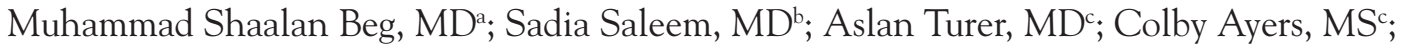 \\ James A. de Lemos, MDc; Amit Khera, MDc; Philipp E. Scherer, PhD'; and Susan G. Lakoski, MD
}

\begin{abstract}
Background: Adiponectin dysregulation is postulated to affect cancer risk via modulation of insulin resistance and inflammation. Epidemiologic studies evaluating this relationship have conflicting results and data from non-white cohorts are lacking. We examined the association between adiponectin and risk of cancer incidence in the multiethnic Dallas Heart Study (DHS). Methods: Participants enrolled in the DHS and known adiponectin values were included. Incident cancer cases were identified through a systematic linkage of the DHS and the Texas Cancer Registry. Univariate/multivariate analysis were performed to test the association between adiponectin and incident cancer after adjusting for age, diabetes status, gender, ethnicity, C-reactive protein level, smoking status, and body mass index. Adiponectin level was evaluated both as a continuous variable and in race/ethnicity specific quartiles. Results: Of 3444 individuals, there were 152 incident cancers. The study population was comprised of $44.4 \%$ men, and $51.05 \%$ were black. Baseline median adiponectin levels were 6.43 mcg/ $\mathrm{mL}$ (interquartile range [IQR], $4.37-9.45 \mathrm{mcg} / \mathrm{mL}$ ) in the incident cancer group versus $6.33 \mathrm{mcg} / \mathrm{mL}$ (IQR, $4.57-9.97 \mathrm{mcg} / \mathrm{mL}$ ) in those without cancer. In multivariable analysis, adiponectin level was not associated with cancer incidence after adjusting for covariates. In analyses stratified by race/ethnic group, no association was observed in white, Hispanic, or African American subgroups. Conclusions: In this study of a predominant ethnic minority population, no association between adiponectin and cancer incidence was demonstrated. Despite preclinical rationale and confirmatory findings in other studies, this association may not replicate across all ethnic populations. Additional studies with strong minority representation are warranted to further examine this association. (J Natl Compr Canc Netw 2015;13:873-878)
\end{abstract}

\section{Background}

Adiponectin, a hormone secreted by adipocytes, has increasingly been recognized as a key regulator of insulin sensitivity and inflammation, with low adiponectin levels associated with hyperinsulinemia and other insulinresistant states. ${ }^{1-3}$ Circulating concentrations of adiponectin are reduced in diabetes mellitus, obesity, and older age, and increase with weight loss, ${ }^{4-6}$ and administration of adiponectin has been shown to improve insulin sensitivity. ${ }^{4-7}$ Dysregulation of adiponectin has been postulated to directly or indirectly contribute toward a higher risk of tumor development with multiple mechanisms proposed for tumorigenesis, including increased

From the aDepartment of Internal Medicine, Division of Hematology/ Oncology, UT Southwestern Medical Center, Dallas; 'bepartment of Breast Medical Oncology, The University of Texas MD Anderson Cancer Center, Houston; 'Department of Internal Medicine, Division of Cardiology, UT Southwestern Medical Center, Dallas, and dDepartment of Internal Medicine, Touchstone Diabetes Center, The University of Texas Southwestern Medical Center, Dallas, Texas; and the eDivision of Hematology/Oncology, Vermont Cancer Center, University of Vermont, Burlington, Vermont.

Submitted October 29, 2014; accepted for publication April 22, 2015. concentrations of tumor necrosis factor- $\alpha$, interleukin- 6 (IL-6), and insulin-like growth factor- $1 .^{8}$ Adiponectin has also been shown to inhibit tumor proliferation and angiogenesis, ${ }^{9}$ whereas other studies have found the opposite result. ${ }^{10,11}$ Given the role of adiponectin in insulin resistance, diabetes, and obesity, low adiponectin levels can be an important biological pathway between obesity and cancer development. ${ }^{12,13}$

Because lifestyle modifications, weight loss, and pharmaceutical interventions with agents, including the peroxisomal proliferator-activated receptor $\gamma$ agonists thiazolidinediones, can modify adiponectin levels, it remains important to establish the role of adiponec-
The authors have disclosed that they have no financial interests, arrangements, affiliations, or commercial interests with the manufacturers of any products discussed in this article or their competitors. This work was supported by grants NIH KL2TR001103, UL1TR001105. Results of this study were presented in part at the ASCO 2013 Annual Meeting, in Chicago, IL.

Correspondence: Muhammad Shaalan Beg, MD, Division of Hematology/ Oncology, UT Southwestern Medical Center, 5323 Harry Hines Boulevard, Dallas, TX 75390-8852.

E-mail: Muhammad.Beg@UTSouthwestern.edu 
Beg et al

tin in carcinogenesis and future cancer incidence. Retrospective studies evaluating the association between adiponectin and cancer may be biased because of reverse causation of cancer weight loss on adiponectin levels. Prospective studies from large datasets have evaluated the association between adiponectin and risk of future cancer occurrence, with conflicting results. ${ }^{14-23}$ However, most of these studies lack sociodemographic diversity, and therefore the results are not readily generalizable.

The Dallas Heart Study (DHS) was established to study ethnic differences in cardiovascular health at the community level, wherein a probability-based sample of Dallas County residents were surveyed with an intentional oversampling of black individuals. This study is uniquely positioned to determine prospectively whether adiponectin levels predict future risk of cancer in a multiethnic cohort of men and women. We explored the role of prediagnostic plasma adiponectin in predicting cancer risk in the DHS.

\section{Methods}

\section{Study Population}

Details on the design and enrollment of the DHS have been previously described. ${ }^{24}$ Briefly, the DHS is a well-characterized single site, multiethnic, population-based probability sample of Dallas County residents aged 18 to 65 years that enrolled participants between July 2000 and January 2002. This cohort has been designed to produce an unbiased population estimate of biologic and social variables, and nonHispanic blacks were intentionally oversampled to compose half of the study population. At study entry, participants completed a detailed survey, objectively measured anthropometry, and laboratory testing. Ethnicity was self-assigned in accordance with US census categories. First, structured interviews and anthropometric and blood pressure measurements were performed in the homes of 6101 individuals aged 18 to 65 years (54\% black). A second home visit was then performed to obtain blood and urine samples. Hypertension was defined as blood pressure of $140 / 90 \mathrm{~mm} \mathrm{Hg}$ or greater or taking antihypertensive medications. Diabetes mellitus was defined as a fasting serum glucose level of $126 \mathrm{mg} / \mathrm{dL}$ or greater, self-reported diabetes, or taking hypoglycemic medication. Smoking was defined as cigarette use within the previous 30 days and a lifetime history of having smoked 100 or more cigarettes. The current study population was composed of cases with available adiponectin levels. The protocol was approved by the Institutional Review Board of the University of Texas Southwestern Medical Center in Dallas. All participants provided written informed consent to participate in the DHS.

The DHS has been systematically linked to the Texas Cancer Registry (TCR) to determine cancer cases in the cohort. The TCR is a population-based registry of the state of Texas, and meets quality data standards of both the National Program of Cancer Registries (CDC) and North American Association of Central Cancer Registries. The Texas Cancer Incidence Reporting Act mandates health care facilities, including hospitals, ambulatory surgical centers, and cancer treatment centers, to report to the TCR. All cancer cases identified by the TCR were classified as "prevalent" or "incident" based on the date of cancer occurrence in relation to the date of enrollment to the DHS. Cases with a date of cancer diagnosis 2 years after their date of enrollment to the DHS were classified as "incident," and the remaining as "prevalent" cases. In cases with more than one known cancer, only the first cancer report was included.

Fasting blood samples were obtained from participants and collected in EDTA-containing tubes on the second DHS visit. Plasma aliquots were stored at $-80^{\circ} \mathrm{C}$. A commercially available sandwich enzyme-linked immunosorbent assay (Millipore, Billerica, MA) was used to quantify total adiponectin levels according to manufacturer's specification. The measured intra-assay coefficients of variation $(\mathrm{CVs})$ were between $1.0 \%$ and $7.4 \%$ and the interassay $\mathrm{CVs}$ between $2.4 \%$ and $8.4 \% .{ }^{25} \mathrm{~A}$ high-sensitivity assay for C-reactive protein (CRP) was performed using the Roche/Hitachi 912 System (Roche Diagnostics, Indianapolis, IN), as described previously. ${ }^{26}$

\section{Statistical Analysis}

Clinical and biochemical data of the study population was reported as proportions or median values with 25 th and 75 th percentiles (interquartile range [IQR]) as appropriate. Adiponectin levels were modeled following a natural logarithmic transformation. Because adiponectin levels are highly dependent on gender and race, gender/race-specific quartiles for the entire population were constructed. Adiponectin quartiles were compared with each tumor type using the Cochran-Armitage trend test. Univariate 
correlations were expressed using the Pearson correlation coefficient for normally distributed parameters and the Spearman correlation coefficient for non-normally distributed data as appropriate. Multivariable cox regression analyses were generated to test the association between the presence of incident cancers and adiponectin (log transformed and quartiles). Models were adjusted for age, diabetes status, gender, ethnicity, CRP level, smoking status, and body mass index. $P$ values of less than .05 were considered statistically significant. Based on our number of cases, for $80 \%$ power, we can detect a relative risk of 1.27. All analyses were performed using SAS version 9.2 (SAS Institute Inc., Cary, NC).

\section{Results}

A total of 3444 participants with known adiponectin levels were included in the analysis, with a median age of 43 years (IQR, 36-51 years). The cohort was 51\% black, 30\% white and 17\% Hispanic, whereas 55.6\% were female. Mean body mass index was $29.6 \mathrm{~kg} / \mathrm{m}^{2}$. Among the cohort, 11.7\% had diabetes mellitus, 29.2\% were smokers, and $33.6 \%$ had a history of hypertension (Table 1). A total of 246 cancer cases were identified from the TCR. Of those, 152 were incident cancers. The total person-years follow-up for this study is 40,988 .

Median adiponectin levels were higher $(P<.001)$ in women $(7.47 \mathrm{mcg} / \mathrm{mL})$ compared with men $(5.53$ $\mathrm{mcg} / \mathrm{mL})$, and whites had a higher adiponectin level than Hispanics and blacks $(8.16 \mathrm{mcg} / \mathrm{mL}$ vs 6.37 and $5.72 \mathrm{mcg} / \mathrm{mL}$, respectively; $P<.001$ ) (Table 2 ).

The median adiponectin level was $6.33 \mathrm{mcg} / \mathrm{mL}$ (IQR, 4.57-9.97) in the cancer group and 6.43 $\mathrm{mcg} / \mathrm{mL}$ (IQR, 4.37-9.45) in the noncancer group $(P=.736)$. Among incident cancers, the most common cancer sites were breast $(32 \%)$, gastrointestinal tract $(24 \%)$, and prostate $(22 \%)$. Median adiponectin levels were $7.65 \mathrm{mcg} / \mathrm{mL}$ (IQR, 5.82-11.11) in breast cancer, $5.94 \mathrm{mcg} / \mathrm{mL}$ (IQR, $4.34 \mathrm{mcg} / \mathrm{mL}$ 8.17 ) in gastrointestinal cancers, and $6.22 \mathrm{mcg} / \mathrm{mL}$ (IQR, 3.31-8.5) in prostate cancer (Table 3).

In the overall population, no univariate association was seen between 1-log difference in adiponectin level and incident cancer $(1.03 ; 95 \% \mathrm{CI}, 0.89$, $1.18 ; P=.73$ ) or after stratifying by gender (men, 1.07 [95\% CI, 0.86, 1.328; $P=.53]$ and women, $0.98[95 \%$ $\mathrm{CI}, 0.81,1.18 ; P=.83])$. An interaction between race/ ethnic group and adiponectin level was observed in the univariable analyses $(P=.02)$. However, stratification by race/ethnic group did not reveal a significant association between adiponectin level and incident cancer in whites (hazard ratio [HR], 1.48; 95\% CI, 0.93, 2.33; $P=.10$ ], Hispanics (HR, 1.04; 95\% CI, $0.47,2.33 ; P=.92$ ), or blacks (odds ratio [OR], 0.84; $95 \% \mathrm{CI}, 0.62,1.13 ; P=.25)$. Similar findings in the overall population and in race/ethnicity strata were demonstrated after adjusting for age, diabetes status,

\begin{tabular}{|c|c|c|c|c|c|}
\hline & $\begin{array}{l}\text { Overall } \\
(\mathrm{N}=3444)\end{array}$ & $\begin{array}{l}\text { Quartile } 1 \\
(\mathrm{~N}=858)\end{array}$ & $\begin{array}{l}\text { Quartile } 2 \\
(\mathrm{~N}=863)\end{array}$ & $\begin{array}{l}\text { Quartile } 3 \\
(\mathrm{~N}=863)\end{array}$ & $\begin{array}{l}\text { Quartile } 4 \\
(\mathrm{~N}=860)\end{array}$ \\
\hline Median age, y (IQR) & $43(36-51)$ & $42(35-50)$ & $44(36-51)$ & $43(36-52)$ & $45(37-52)$ \\
\hline $\begin{array}{l}\text { Median adiponectin, } \\
\mathrm{mcg} / \mathrm{mL} \text { (IQR) }\end{array}$ & $6.43(4.37-9.46)$ & $3.27(2.50-4.10)$ & $5.21(4.59-6.13)$ & $7.69(6.55-8.86)$ & 11.85 (9.93-14.84) \\
\hline Mean BMI, kg/m² (SD) & $29.60(7.02)$ & $31.77(7.18)$ & $30.69(6.92)$ & $29.09(6.77)$ & $26.82(6.19)$ \\
\hline \multicolumn{6}{|l|}{ Race } \\
\hline Black & $1758(51.05 \%)$ & $438(51.05 \%)$ & $440(50.98 \%)$ & $440(50.98 \%)$ & $440(51.16 \%)$ \\
\hline White & $1018(29.56 \%)$ & $254(29.60 \%)$ & $255(29.55 \%)$ & $255(29.55 \%)$ & $254(29.53 \%)$ \\
\hline Hispanic & $594(17.25 \%)$ & $148(17.25 \%)$ & $149(17.27 \%)$ & $149(17.27 \%)$ & $148(17.21 \%)$ \\
\hline Other & $74(2.15 \%)$ & $18(2.10 \%)$ & $19(2.02 \%)$ & $19(2.20 \%)$ & $18(2.09 \%)$ \\
\hline Male & $1529(44.4 \%)$ & $381(44.41 \%)$ & $383(44.38 \%)$ & $383(44.38 \%)$ & $382(44.42 \%)$ \\
\hline Diabetes & $402(11.68 \%)$ & $153(17.83 \%)$ & $96(11.12 \%)$ & $84(9.74 \%)$ & $69(8.02 \%)$ \\
\hline Hypertension & $1140(33.6 \%)$ & $312(36.66 \%)$ & $297(35.23 \%)$ & $260(30.44 \%)$ & $271(32.07 \%)$ \\
\hline Smoking & $1003(29.17 \%)$ & $257(29.95 \%)$ & $242(28.04 \%)$ & $244(28.31 \%)$ & $260(30.37 \%)$ \\
\hline
\end{tabular}

Abbreviations: BMI, body mass index; IQR, interquartile range. 
Beg et al

\begin{tabular}{|c|c|c|}
\hline & $\begin{array}{l}\text { Median Adiponectin, } \\
\mathrm{mcg} / \mathrm{mL} \\
\text { (IQR) }\end{array}$ & $P$ Value \\
\hline Sex & & $<.001$ \\
\hline Female & $7.47(4.92-10.71)$ & \\
\hline Male & $5.53(3.80-8.05)$ & \\
\hline Race/ethnicity & & $<.001$ \\
\hline Black & $5.72(3.96-8.50)$ & \\
\hline White & $8.16(5.47-11.55)$ & \\
\hline Hispanic & $6.37(4.48-8.85)$ & \\
\hline
\end{tabular}

Abbreviation: IQR, interquartile range.

gender, ethnicity, CRP level, smoking status, and BMI, and on categorizing adiponectin into quartiles (Table 4). Moreover, no interaction by race/ethnicity was observed in the multivariable adjusted analyses $(P=.142)$. We performed analysis based on follow-up years ( $2-5$ years, $5-10$ years, and $>10$ years) to help define the impact of exposure window on the relationship, and no association was found. Additionally, no association was found when comparing incident cancer between the 5 th percentile and 95 th percentile adiponectin levels $(P=.81)$.

\section{Discussion}

Despite strong preclinical rationale, previous epidemiologic studies evaluating the association of incident cancer and adiponectin levels have had conflicting results. This analysis from a large multiethnic population-based cohort has demonstrated

\begin{tabular}{|c|c|c|}
\hline & $\mathbf{N}$ & $\begin{array}{l}\text { Median Adiponectin, } \\
\mathrm{mcg} / \mathrm{mL} \\
(\mathrm{IQR})\end{array}$ \\
\hline Incident cancer & 152 & 6.33 (4.57-9.97) \\
\hline Breast & 49 & $7.65(5.82-11.11)$ \\
\hline Gastrointestinal & 36 & $5.94(4.34-8.17)$ \\
\hline Prostate & 34 & $6.22(3.31-8.50)$ \\
\hline Lung & 17 & $6.13(4.70-8.49)$ \\
\hline Gynecologic & 16 & $5.99(3.89-11.48)$ \\
\hline No cancer & 3292 & $6.43(4.37-9.45)$ \\
\hline
\end{tabular}

Abbreviation: IQR, interquartile range. that adiponectin level is not associated with future cancer incidence in the overall population-based DHS sample.

A large nested case-control study of 468 pancreatic cancer case subjects and 1080 matched control subjects from 5 prospective US cohorts showed that plasma adiponectin level was inversely associated with pancreatic cancer risk, which was consistent across the 5 prospective cohorts and independent of other markers of insulin resistance (eg, diabetes, BMI, physical activity, plasma C-peptide). ${ }^{14}$ Prospective case-control studies have suggested that low plasma adiponectin concentrations ${ }^{16}$ and high-density lipoprotein cholesterol/adiponectin ratio ${ }^{15}$ are associated with colon cancer risk. A study nested in a cohort of Finnish male smokers evaluated the relationship between prediagnostic serum adiponectin level and renal cell carcinoma (RCC) risk and showed an association between high adiponectin levels and reduced RCC risk after adjustment for BMI. ${ }^{17}$

However, other studies exploring this association have not confirmed these findings. Another study from the EPIC cohort demonstrated an association between markers of inflammation and hyperinsulinemia, including CRP, IL-6, C-peptide, and non-high-molecular-weight adiponectin, and an increased risk of hepatocellular cancer (HCC) independent of obesity and historically established risk factors for HCC. ${ }^{18}$ Whereas the panel of markers listed in the prior discussion did increase the predictive potential of the model, when looked at individually among the markers examined, high - not low-levels of adiponectin were predictive, which does not fully support the proposed hypothesis. Tworoger et $\mathrm{al}^{21}$ performed a nested case-control study within the Nurses' Health Study (NHS) and demonstrated an inverse relationship between adiponectin level and breast cancer risk in postmenopausal women but no relationship among premenopausal women. However, the association in postmenopausal women was not replicated in 2 other prospective case-control studies nested in the Multiethnic Cohort and the Cancer Prevention Study II (CPS-II). ${ }^{22,23}$ No association was detected between prediagnostic serum total adiponectin or high-molecular-weight adiponectin levels and endometrial cancer risk in a nested case-control study within the Breast and Bone Follow-up to the Fracture Intervention Trial (B FIT; $\mathrm{n}=15,595) .{ }^{19} \mathrm{How}$ - 
Adiponectin and Cancer Incidence

\begin{tabular}{|c|c|c|c|c|}
\hline & $\begin{array}{l}\text { Overall } \\
(\mathrm{N}=3444)\end{array}$ & $\begin{array}{l}\text { Black } \\
(\mathrm{N}=1758)\end{array}$ & $\begin{array}{l}\text { White } \\
(\mathrm{N}=1018)\end{array}$ & $\begin{array}{l}\text { Hispanic } \\
(\mathrm{N}=594)\end{array}$ \\
\hline \multicolumn{5}{|c|}{ Distribution of cancer cases $(n=152)$} \\
\hline Quartile 1, n (PY) & $37(10,118)$ & $24(5132)$ & $5(3035)$ & $5(1746)$ \\
\hline Quartile 2, n (PY) & $42(10,248)$ & $23(5217)$ & $12(3048)$ & $7(1756)$ \\
\hline Quartile 3, n (PY) & $37(10,342)$ & $18(5262)$ & $18(3046)$ & $0(1809)$ \\
\hline Quartile 4, n (PY) & $36(10,280)$ & $19(5281)$ & $12(3024)$ & $3(1763)$ \\
\hline \multicolumn{5}{|l|}{ Univariate } \\
\hline Adiponectin (log) & $1.03(0.81,1.30)$ & $0.84(0.62,1.13)$ & $1.48(0.93,2.33)$ & $1.04(0.47,2.33)$ \\
\hline \multicolumn{5}{|c|}{ Adiponectin quartiles } \\
\hline 1 (referent) & 1 & 1 & 1 & 1 \\
\hline 2 & $1.14(0.78,1.67)$ & $1.02(0.62,1.67)$ & $1.78(0.82,3.85)$ & $1.31(0.45,3.78)$ \\
\hline 3 & $0.96(0.64,1.42)$ & $0.76(0.44,1.29)$ & $2.65(1.79,4.76)$ & $0.16(0.02,1.32)$ \\
\hline 4 & $1.02(0.69,1.57)$ & $0.82(0.49,1.38)$ & $1.79(0.83,3.88)$ & $0.97(0.31,3.03)$ \\
\hline \multicolumn{5}{|l|}{ Multivariable } \\
\hline Adiponectin (log) & $1.03(0.80,1.32)$ & $0.89(0.65,1.24)$ & $1.43(0.85,2.43)$ & $0.68(0.30,1.52)$ \\
\hline \multicolumn{5}{|c|}{ Adiponectin quartiles } \\
\hline 1 (referent) & 1 & 1 & 1 & 1 \\
\hline 2 & $1.18(0.81,1.74)$ & $1.07(0.65,1.76)$ & $1.64(0.75,3.57)$ & $1.20(0.41,3.49)$ \\
\hline 3 & $1.02(0.68,1.53)$ & $0.87(0.51,1.50)$ & $1.90(0.89,4.09)$ & $0.14(0.02,1.17)$ \\
\hline 4 & $1.05(0.67,1.50)$ & $0.92(0.53,1.60)$ & $1.53(0.68,3.42)$ & $0.65(0.20,2.13)$ \\
\hline
\end{tabular}

Abbreviation: PY, person years.

ever, an analysis of the EPIC study did demonstrate the relationship between lower prediagnostic plasma adiponectin concentrations and a higher risk of endometrial cancer in both premenopausal and postmenopausal women, regardless of BMI status. ${ }^{20}$

The studies discussed herein evaluating adiponectin all lack racial and socioeconomic diversity, which limits the generalizability of these findings. An exception is the Multiethnic Cohort, which only evaluated postmenopausal breast cancer risk, and similar to our analysis, did not detect an association between cancer and adiponectin level. ${ }^{23}$ Notably, the composition of the Multiethnic Cohort study is largely Japanese American women (35\%), and therefore distinct from the DHS population.

The study design used for the present evaluation had some limitations. DHS has been systematically linked to the TCR to determine incident cancer cases in the cohort, and a cutoff of 2 years between enrollment in the DHS and date of cancer diagnosis was used to separate "incident" from "prevalent" cancer cases. This time point was chosen as a reasonable period wherein if a case had an undiagnosed malignancy at the time of enrollment to DHS, sufficient time would lapse to allow the malignancy to clinically manifest. By placing the cutoff mark at 2 years we minimized the effect of subclinical or undiagnosed "prevalent" cancer cases from confounding adiponectin levels, but this reduced the number of cancer cases and diminished the power to detect a statistically significant difference. Patients enrolled in the DHS and whose cancer may have been diagnosed outside the state of Texas may have not been captured. Adiponectin levels are highly associated with ethnicity and gender, and previous studies largely lack data on ethnic minorities. The intentional oversampling of the black population in the DHS cohort provides the opportunity to evaluate the association in ethnic minorities who are not well represented in other cohorts. Single nucleotide polymorphism (SNP) adiponectin and adiponectin receptor 1 are gaining recognition as being associated with cancer and may provide further insight into this relationship, particularly in reference to potential ethnic differences. ${ }^{27,28}$ SNP analysis was not performed as part of this study. The rigorous DHS 
study design, very well-defined enrollment characteristics, and high rate of serum samples are particular strengths of this study. Therefore, this study is uniquely positioned to determine prospectively whether adiponectin levels predict future risk of cancer in a multiethnic cohort of men and women.

Despite preclinical rationale, our analysis of the multiethnic DHS does not support an association between prediagnostic adiponectin levels and future cancer risk. Analysis from other cohorts evaluating the association of adiponectin level and individual cancers types have not had consistent findings and have lacked ethnic diversity, which limits the broad applicability of those findings. Further studies are necessary to understand the mechanism of carcinogenesis in obesity and insulin resistance in a prospective manner.

\section{References}

1. Cnop M, Havel PJ, Utzschneider KM, et al. Relationship of adiponectin to body fat distribution, insulin sensitivity and plasma lipoproteins: evidence for independent roles of age and sex. Diabetologia 2003;46:459-469.

2. Pajvani UB, Scherer PE. Adiponectin: systemic contributor to insulin sensitivity. Curr Diab Rep 2003;3:207-213.

3. Wolf AM, Wolf D, Rumpold H, et al. Adiponectin induces the antiinflammatory cytokines IL-10 and IL-1RA in human leukocytes. Biochem Biophys Res Commun 2004;323:630-635.

4. Gavrila A, Chan JL, Yiannakouris N, et al. Serum adiponectin levels are inversely associated with overall and central fat distribution but are not directly regulated by acute fasting or leptin administration in humans: cross-sectional and interventional studies. J Clin Endocrinol Metab 2003;88:4823-4831.

5. Arita Y, Kihara S, Ouchi N, et al. Paradoxical decrease of an adiposespecific protein, adiponectin, in obesity. Biochem Biophys Res Commun 1999;257:79-83

6. Hoffstedt J, Arvidsson E, Sjolin E, et al. Adipose tissue adiponectin production and adiponectin serum concentration in human obesity and insulin resistance. J Clin Endocrinol Metab 2004;89:1391-1396.

7. Trujillo ME, Scherer PE. Adiponectin-journey from an adipocyte secretory protein to biomarker of the metabolic syndrome. J Intern Med 2005;257:167-175

8. Kanazawa I, Yamaguchi T, Sugimoto T. Serum insulin-like growth factor-I is negatively associated with serum adiponectin in type 2 diabetes mellitus. Growth Horm IGF Res 2011;21:268-271.

9. Brakenhielm E, Veitonmaki N, Cao R, et al. Adiponectin-induced antiangiogenesis and antitumor activity involve caspase-mediated endothelial cell apoptosis. Proc Natl Acad Sci U S A 2004;101:2476-2481.
10. Landskroner-Eiger S, Qian B, Muise ES, et al. Proangiogenic contribution of adiponectin toward mammary tumor growth in vivo. Clin Cancer Res 2009; 15:3265-3276.

11. Denzel MS, Hebbard LW, Shostak G, et al. Adiponectin deficiency limits tumor vascularization in the MMTV-PyV-mT mouse model of mammary cancer. Clin Cancer Res 2009;15:3256-3264.

12. Kadowaki T, Yamauchi T, Kubota N, et al. Adiponectin and adiponectin receptors in obesity-linked insulin resistance. Novartis Found Symp 2007;286:164-176; discussion 176-182, 200-203.

13. Wolin KY, Carson K, Colditz GA. Obesity and cancer. Oncologist 2010;15:556-565.

14. Bao Y, Giovannucci EL, Kraft P, et al. A prospective study of plasma adiponectin and pancreatic cancer risk in five US cohorts. J Natl Cancer Inst 2013;105:95-103.

15. Aleksandrova $K$, Boeing $H$, Jenab $M$, et al. Total and high-molecular weight adiponectin and risk of colorectal cancer: the European Prospective Investigation into Cancer and Nutrition Study. Carcinogenesis 2012;33:1211-1218.

16. Wei EK, Giovannucci E, Fuchs CS, et al. Low plasma adiponectin levels and risk of colorectal cancer in men: a prospective study. J Natl Cancer Inst 2005;97:1688-1694.

17. Liao LM, Weinstein SJ, Pollak M, et al. Prediagnostic circulating adipokine concentrations and risk of renal cell carcinoma in male smokers. Carcinogenesis 2013;34:109-112.

18. Aleksandrova $K$, Boeing $H$, Nothlings $U$, et al. Inflammatory and metabolic biomarkers and risk of liver and bilary tract cancer. Hepatology 2014;60:858-871.

19. Dallal CM, Brinton LA, Bauer DC, et al. Obesity-related hormones and endometrial cancer among postmenopausal women: a nested case-control study within the B FIT cohort. Endocr Relat Cancer 2013;20:151-160.

20. Cust AE, Kaaks R, Friedenreich $C$, et al. Plasma adiponectin levels and endometrial cancer risk in pre- and postmenopausal women. J Clin Endocrinol Metab 2007;92:255-263.

21. Tworoger SS, Eliassen $A H$, Kelesidis $T$, et al. Plasma adiponectin concentrations and risk of incident breast cancer. J Clin Endocrinol Metab 2007;92:1510-1516.

22. Gaudet MM, Patel AV, Teras LR, et al. Obesity-related markers and breast cancer in CPS-II Nutrition Cohort. Int J Mol Epidemiol Genet 2013;4:156-166.

23. Ollberding NJ, Kim Y, Shvetsov YB, et al. Prediagnostic leptin adiponectin, C-reactive protein, and the risk of postmenopausal breast cancer. Cancer Prev Res (Phila) 2013;6:188-195.

24. Victor RG, Haley RW, Willett DL, et al. The Dallas Heart Study: a population-based probability sample for the multidisciplinary study of ethnic differences in cardiovascular health. Am J Cardiol 2004;93:14731480 .

25. Turer AT, Khera A, Ayers CR, et al. Adipose tissue mass and location affect circulating adiponectin levels. Diabetologia 2011;54:2515-2524.

26. Abdullah SM, Khera A, Leonard D, et al. Sex differences in the association between leptin and CRP: results from the Dallas Heart Study. Atherosclerosis 2007;195:404-410

27. Kaklamani VG, Hoffmann TJ, Thornton TA, et al. Adiponectin pathway polymorphisms and risk of breast cancer in African Americans and Hispanics in the Women's Health Initiative. Breast Cancer Res Treat 2013;139:461-468.

28. Kaklamani V, Yi N, Zhang $\mathrm{K}$, et al. Polymorphisms of ADIPOQ and ADIPOR1 and prostate cancer risk. Metabolism 2011;60:1234-1243. 